\title{
The Architecture of a Software System for Supporting Community-based Primary Health Care with Mobile Technology: The Mobile Technology for Community Health (MoTeCH) Initiative in Ghana
}

\author{
Bruce MacLeod ${ }^{1}$, James Phillips ${ }^{2}$, Allison E. Stone², Aliya Walji ${ }^{3}$, \\ John Koku Awoonor-Williams 4
}

${ }^{1}$ Mailman School of Public Health, Columbia University, New York, NY and Computer Science Department at the University of Southern Maine, Portland, Maine

${ }^{2}$ Mailman School of Public Health, Columbia University, New York, NY

${ }^{3}$ Grameen Foundation, Seattle WA

${ }^{4}$ Upper East Regional Director of Health Services, Ghana Health Service

\section{Abstract}

This paper describes the software architecture of a system designed in response to the health development potential of two concomitant trends in poor countries: i) The rapid expansion of community health worker deployment, now estimated to involve over a million workers in Africa and Asia, and ii) the global proliferation of mobile technology coverage and use. Known as the Mobile Technology for Community Health (MoTeCH) Initiative, our system adapts and integrates existing software applications for mobile data collection, electronic medical records, and interactive voice response to bridge health information gaps in rural Africa. MoTeCH calculates the upcoming schedule of care for each client and, when care is due, notifies the client and community health workers responsible for that client. MoTeCH also automates the aggregation of health status and health service delivery information for routine reports. The paper concludes with a summary of lessons learned and future system development needs.

Key words: Maternal, Child Health, Electronic Medical Records, Mobile Phones, Low Resource Settings

\section{Introduction}

Mounting evidence that community-based primary health care can improve the survival of mothers and children have fostered international commitment to expand community health programs in poor countries ${ }^{1}$. Over a million paid community health workers now work in village 
The Architecture of a Software System for Supporting Community-based Primary Health Care with Mobile Technology: The Mobile Technology for Community Health (MoTeCH) Initiative in Ghana

locations in Asia and Africa ${ }^{2}$. This trend coincides with an acceleration of access to mobile telephones, currently estimated to number in the range of five billion globally ${ }^{3}$. These simultaneous developments have generated international interest in potential synergies that could improve the quality and coverage of primary health care. In 2009, Columbia University, the Grameen Foundation, and the Ghana Health Service responded to this opportunity by launching a program of technology development and research designed to evaluate the potential uses of mobile technology in supporting community health operations. Known as the "Mobile Technology for Community Health" (MoTeCH) Initiative, the project focuses on leveraging mobile phone technologies to improve the health of the pregnant women and young children in the Upper East Region of Ghana -an impoverished and isolated locality where childhood tropical diseases such as malaria are hyper-endemic, mortality is generally high, and risks associated with childbirth are extreme. This paper describes the context that motivated the design of $\mathrm{MoTeCH}$ and the software architecture of a pilot system that is now in operation in one district in northern Ghana.

\section{The problem}

High quality and timely health information is not readily available to pregnant women and new mothers in the Upper East Region. Internet access is virtually non-existent, literacy rates are low, and travel distances to clinics can be significant for women. The need for quality health information in the Upper East region is especially important. Local beliefs and superstitions oftentimes guide women where there are no other information alternatives reflecting international norms for best practices ${ }^{4}$. For example, in Kassena-Nankana West, elder female relatives encourage younger pregnant women to labor at home for as long as they can stand before going to a health facility, as this is a mark of strength and willpower ${ }^{5}$. For these reasons and others, Ghana Health service encourages women to visit local clinics at least four times before delivery. In the women's first visit to the clinic, they are given a small paper booklet that provides basic health information for the pregnancy and includes a schedule of upcoming care. Unfortunately, in many cases the woman is simply unable to access the information in this booklet because she is illiterate. Information about infectious diseases is limited, even for conditions that are hyper endemic. In particular, malaria remains the major cause of death among under-5 children, despite low cost and effective means of preventing and curing illness ${ }^{6,7}$. Moreover, the proper use of intermittent preventative treatment during pregnancy (IPTp) can reduce maternal anemia, fetal loss, and dangerous complications for the unborn child.

Frontline health workers in Ghana use five large 11x17 inch notebooks, termed "registers" by the GHS, for recording health service delivery information. The daily register maintains basic information about the patient, date, and the nature of the visit. There are also registers for more detailed care information about antenatal care (ANC) for pregnant mothers, postnatal care (PNC) for young children, and family planning.

The proper use of these registers can be compromised in a number of ways. When frontline health workers are working outside the clinic, we found that many nurses use their own, smaller, notebooks to record information. This data from the community outreach sessions is then transferred to the registers on their return to the clinic and it is not surprising to expect problems in linking the individual level data from the two sources. When a register is full and cannot hold 
The Architecture of a Software System for Supporting Community-based Primary Health Care with Mobile Technology: The Mobile Technology for Community Health (MoTeCH) Initiative in Ghana

additional information, the health worker is instructed to transfer information regarding currently active patients into a new register, creating the possibility of inaccurate transfers or missing information. Nurses are expected to determine when clients do not meet the recommended appointments for routine antenatal and postnatal care. Determining these lapses in care would require regular care schedule calculations for each client and this work is not always done due to low prioritization, inadequate training, or insufficient supervision. Finally, the proper linkage of longitudinal care information for a patient can be challenging when different frontline health workers, such as nurses and midwifes, both access and update information in the registers, and patient identification is not always obvious.

Health administrators and planners benefit from a clear and accurate picture of health service delivery operations and the health status of the population. To obtain this information, nurses in Ghana are asked to summarize ANC, PNC, family planning, morbidity, and mortality counts by gender and by age group. This data reporting activity usually requires around three days of work every month. . The quality of the data reported can be easily compromised by not recording all health service delivery or not performing the correct calculations. More fundamentally, this data management task is not central to the routine operations of the nurse and it can be delayed when the nurse prioritizes patient care over the reports.

All of this combines to create information needs and bottlenecks for all participants in the health system (Figure 1). In particular,

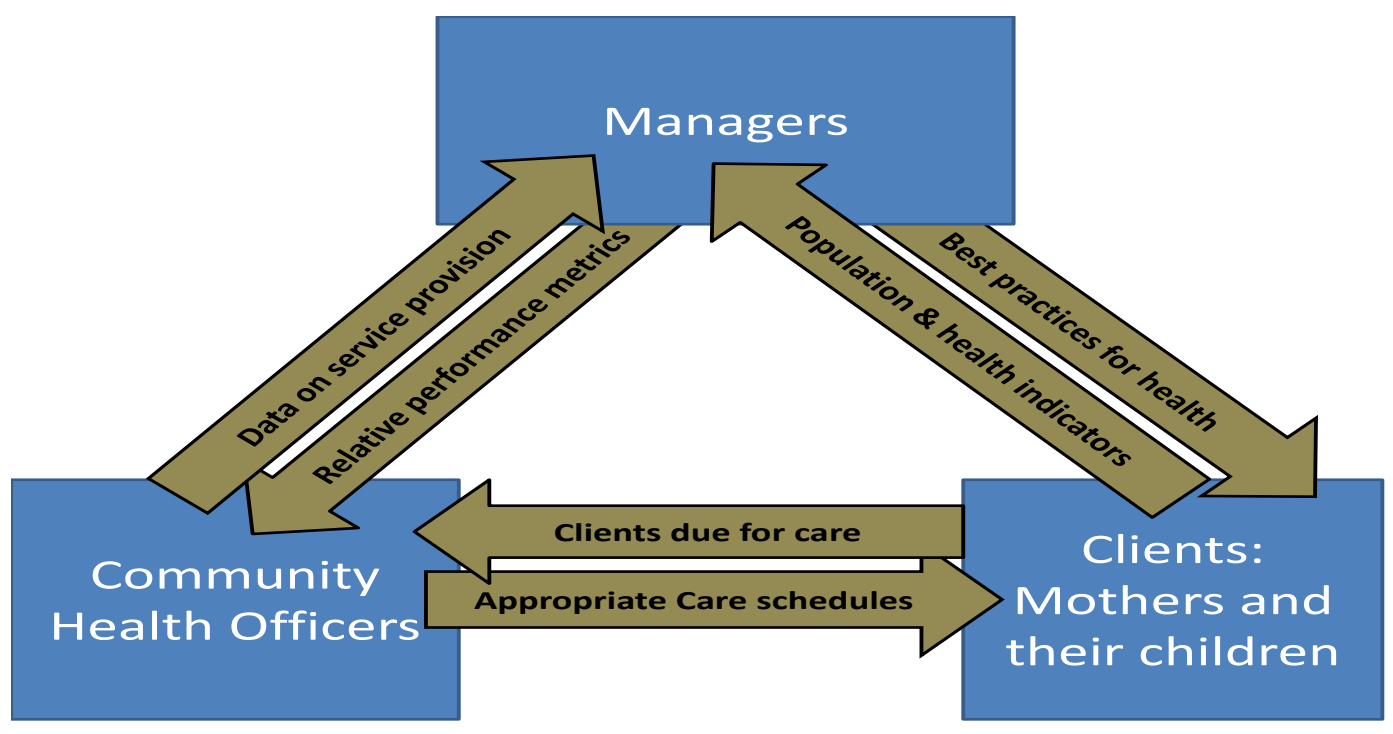

Figure 1: Health Information Needs and Wants 
The Architecture of a Software System for Supporting Community-based Primary Health Care with Mobile Technology: The Mobile Technology for Community Health (MoTeCH) Initiative in Ghana

- Clients seek information to guide health behavior, but clinics can be remote and accessing them detracts from farming or domestic work responsibilities. In addition, health workers can be preoccupied with clinical duties with little time for household outreach. Clients may not be conversant with English and workers may not understand local languages and this linguistic divide can impede the flow of essential health information.

- Frontline health workers use paper based registers to document health service delivery. Ideally, nurses should follow up on patients who do not meet their appointment schedule, but workers often either 1) Are not trained adequately to identify defaulters in their registers; 2) Neglect this work due to lack of time or motivation; or 3) lack supervisory support and oversight in this work.

- Health administrators seek information on the health status of the population and the extent of front line health operations. While each nurse will aggregate service delivery and morbidity information from the paper registers every month, this involves calculations for hundreds of different data points because data needs to be reported by age group, gender and the type of service delivery or disease. The quality and timeliness of the reports can be compromised as a result.

\section{The Context}

The MoTeCH pilot is located in one district of the Upper East Region, known as KassenaNankana West, a locality on Ghana's border with Burkina Faso. In 2010, the population of the district was approximately 74,000. The Ghana Statistical Service ranks the Upper East Region as the poorest of the 10 regions in the country and the most rural ( 84 percent). The KassenaNankana West district where MoTeCH is under trial remains isolated, rural, and remote. The practice of traditional religion is widespread; rites, rituals, and beliefs tend to reinforce and sustain institutions of patriarchy, lineage, and male dominance ${ }^{8,9}$. A strong cultural emphasis on the lineage, in turn, emphasizes the role of the corporate extended family in arranging marriage for the purpose of perpetuating the lineage. Over one half of married women were in polygamous unions in the baseline period.

Geography and human ecology of the area accentuate social isolation and complicate efforts to organize health and human services in the locality. The settlement pattern is highly dispersed, with no towns, modern markets, or industries that would develop centers of trade and communication. There are three primary languages in Kassena-Nankana West District and two of three languages have no established written form. Most of the populations are Kassim or Nankam speaking - languages that provide only fragmentary communication links to Ghana's southern cultures, restricting exposure of the population to outside ideas more generally. Although female literacy is increasing, the rate was only 13 percent in the 1990s.

Health conditions are known to be improving, owing to the successful introduction of community-based primary health care in 1996 by a project of the Navrongo Health Research 
The Architecture of a Software System for Supporting Community-based Primary Health Care with Mobile Technology: The Mobile Technology for Community Health (MoTeCH) Initiative in Ghana

Centre. Termed the "Community-based Health Planning and Services" (CHPS) Initiative, this program provides free basic health care for pregnant women and young children in community and doorstep services that include ANC services such as tetanus injections, anti-malarial medications (IPTi) and iron supplements. CHPS has been an important source of primary health care in the UER because the region generally is lacking medical doctors, with substantially less than the national average of 1 doctor per 8500 people. As in most of Ghana, trained nurses deliver much of the routine health care. In response to the critical need to address manpower shortages and service accessibility problems, CHPS places nurses in village posts to provide primary care where they are known as Community Health Officers (CHOs). They provide care to small catchment areas consisting of a few thousand individuals known as "CHPS zones."

While the Navrongo project resulted in steep declines in child and maternal mortality in the $1990 \mathrm{~s}^{10}$, evidence suggests that its mode of operation has been changing as it has been scaled up in recent years. Immediately prior to the introduction of $\mathrm{MoTeCH}$, baseline research found many $\mathrm{CHOs}$ were not placing a priority on community outreach, but were instead waiting for clients to come to their facilities ${ }^{11}$. One of MoTeCH's premises is the hypothesis that as CHPS developed a focus on the construction of health posts, supervisory attention on household services waned in the decade following 2000, and eroding emphasis was placed on community outreach activities $^{12}$.

During this period, cell phone coverage for this rural, agrarian, society has been expanding rapidly. As in much of Africa, the lack of investment in fixed line telecommunications has allowed the companies to quickly introduce advanced wireless communication and fill an immediate need for communication services ${ }^{13,14}$. In this region, the telecommunications networks support the GPRS data service in most areas which allows basic communication between cell phones and internet servers ${ }^{15}$.

Baseline research found that in Kassena-Nankana West, 23 percent of women of reproductive age personally own a phone; 33 percent have spouses that own a phone; 15 percent have access to a phone owned by other members of their family compound; and 12 percent said they could access a phone in their community if they need to. Only 16 percent of women said they had no access to a mobile phone. Frequency of cell phone use varies significantly with type of phone access. More than 95 percent of respondents with personal access used their phones at least weekly vs. 40 percent of women with spousal access to a phone, 25 percent of women with access in their compound and 12 percent of women with community access.

\section{Methods}

\section{Bridging the Health Information Gaps}

MoTeCH integrates the mobile phone into the rural health system to bridge key health information gaps. Pregnant women and new mothers who own a phone or have access to a phone can get relevant, personalized health information from $\mathrm{MoTeCH}$. Nurses are given a low cost mobile phone (less than \$50) to enter data and receive MoTeCH system generated messages about patients who are overdue for routine health care. The data entered by the nurse is also used to automate the generation of reports for district and regional health administrators and 
The Architecture of a Software System for Supporting Community-based Primary Health Care with Mobile Technology: The Mobile Technology for Community Health (MoTeCH) Initiative in Ghana

planners. Some of the particular usage scenarios from the perspective of the key MoTeCH target groups include:

- Clientele: Pregnant women and caregivers of children under one year can register to receive messages from $\mathrm{MoTeCH}$. Once registered, they receive weekly health educational voice messages (in their local language) that correspond to the gestational age of their pregnancy or the age of their child. For example, a woman in early pregnancy might receive a message explaining that nausea and/or vomiting is expected and normal during this stage. If the client is due for scheduled care, then an early notification of the upcoming clinic visit is included with their weekly educational message. If a patient misses a phone call, or if they do not have regular access to a phone, they can "flash" the system and they will be immediately called back. Then, by entering their unique MoTeCH ID number, they can retrieve their personal messages.

- Frontline health workers: Nurses send patient data to the MoTeCH system using data collection software that operates on low cost mobile phones. Currently, there are sixteen different types of data entry forms pertaining to various client encounters, such as patient registration, antenatal care visits, postnatal care visits, and delivery information. The mobile phone application also includes various query forms that allow real-time retrieval of information regarding clients who are overdue for particular services in the nurses' CHPS zone. Nurses receive a weekly SMS message from $\mathrm{MoTeCH}$ about clients who are overdue for routine scheduled care. This includes a list of any pregnant women that have missed antenatal care visits and children that are overdue for receiving specific immunizations. With these reminders, $\mathrm{MoTeCH}$ makes information about clients in need of care readily available to health workers, with the aim of increasing their outreach to provide these services. Community health volunteers also have a role to play in $\mathrm{MoTeCH}$ : when they become aware that a home birth has occurred in the village, they can contact the MoTeCH call center to report the delivery. Once MoTeCH is notified of a birth, it will send an SMS message every six hours to the health worker responsible for the community where the birth occurred to ensure postnatal care is promptly provided. This alert is repeatedly sent until the nurse sends detailed information about the birth and provision of postnatal care to the system, after traveling to the village to see the new child.

- Health Administrators and Planners: The data captured by MoTeCH (via mobile phonebased entry by health workers) is aggregated to generate the monthly reports that are submitted to district health managers. These reports are normally produced by health workers scanning their paper registers, keeping a tally of health services provided throughout the month, and writing the reports by hand. In $\mathrm{MoTeCH}$, if nurses accurately capture all of their health services data in the $\mathrm{MoTeCH}$ phone, then they can use the monthly reports generated by the $\mathrm{MoTeCH}$ system rather than completing these monthly reporting forms by hand. Health administrators and planners now receive reports that are generated from information that is used as part of routine health service operations. In addition, the regular inflow of patient data has allowed for the development of new types of reports that give a real-time monitoring of the health service delivery activities in rural locations. 
The Architecture of a Software System for Supporting Community-based Primary Health Care with Mobile Technology: The Mobile Technology for Community Health (MoTeCH) Initiative in Ghana

For $\mathrm{MoTeCH}$ to address all of these concerns, its design and implementation requires nothing less than a systemic change in Ghana's existing health information system process (Figure 2). New ways of conveying best health practices to clients are needed, the burden of paper-based reporting should be reduced, and the information systems support for nurses needs to be strengthened. These factors are interrelated, so solving only a piece of the problem risks developing an approach that will not scale and misses key opportunities for synergy. An approach that jointly addresses the health information needs of clients, health workers, managers and policy makers is poised to make the best use of limited resources and have the greatest potential for sustainable results. As Figure 2 illustrates, data capture is managed by front line workers who have limited capacity mobile phones that update a server database. The server, in turn, supports workers and mothers with alerts and reminders, and feeds back reports to workers, supervisors, managers and policy-makers, mechanizing previously cumbersome capabilities for information to support services, supervision, management, and planning. Thus, the central goal of $\mathrm{MoTeCH}$ is to transform Health Management Information System operations from a data extraction operation to an information support system for all key stakeholders in Ghana Health Service operations.

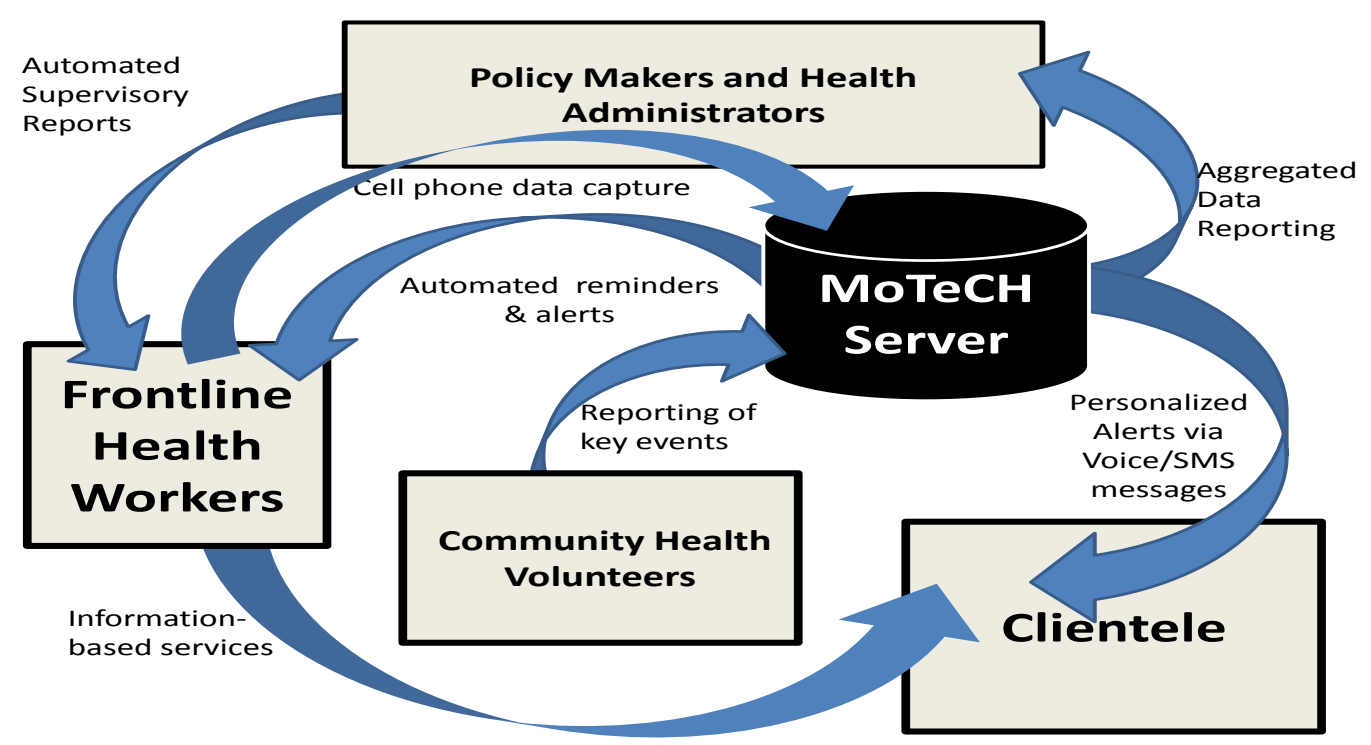

Figure 2: MoTeCH as system for addressing information needs of workers and mothers

While there are many health priorities in this region, the project made the strategic decision to focus on the needs of individuals who are most associated with the unaddressed burden of disease in this setting: i) pregnant women or young mothers, ii) women at times of delivery, a majority of whom are delivering at home, iii) neonates, particularly those requiring outreach in the first 48 hours of life, and iv)children less than one year of age who require immunization and curative health services. Given the significant levels of maternal and child mortality in the Upper East and also that the health care needs of these groups are frequent and time-sensitive, improved information for health workers and clients in these groups holds the most potential for increasing positive health outcomes. Further, $\mathrm{MoTeCH}$ aims to accumulate knowledge from the 
The Architecture of a Software System for Supporting Community-based Primary Health Care with Mobile Technology: The Mobile Technology for Community Health (MoTeCH) Initiative in Ghana

development and deployment of this system that can be used for other health problems and in other low income countries.

\section{The MoTeCH System Architecture}

The MoTeCH system architecture evolved from a strategic decision to build on top-tier software applications that have been field tested elsewhere in Africa. The mobile phone data collection system is based on openXData (www.openxdata.org). This innovative, open source, software application implements a significant portion of the W3C $\mathrm{xForm}$ standard (http://www.w3.org/MarkUp/Forms/) with a relatively small memory footprint on the mobile phone. As a result, we were able to provide the nurses with relatively low cost $(<\$ 50)$ phones and the software development team was able to quickly design and deploy data entry forms. $\mathrm{MoTeCH}$ maintains health information about women and children in the OpenMRS medical record software application (www.openmrs.org). The unique extensible features of OpenMRS allowed us to quickly develop an appropriate data model and to layer project specific software in the medical record application. Finally, the task of delivering voice messages to patients was provided by the IntelliIVR (www.yo.co.ug) application. The Ugandan based company that developed and supports this Interactive-Voice-Response (IVR) application provided expertise in setting up and adapting to the telecommunications situation in Ghana.

Each of these applications brings considerable value to $\mathrm{MoTeCH}$ and a significant amount of project risk was eliminated because these systems have undergone multiple iterations of deployment and revisions in environments similar to those in north-eastern Ghana.

The electronic medical record for each women and child is a central element in the MoTeCH architecture. Information about the health status and service delivery of each client is maintained in an electronic health record. This includes pregnancy due date, visits to the clinic, anti-malarial drugs and childhood immunizations. With this individual health record, $\mathrm{MoTeCH}$ calculates the upcoming schedule of care for each client and, when care is due, it notifies the client and the health workers responsible for that client. The individual level data also addresses the problem of constructing aggregate level health statistics. The onerous, monthly task for each nurse of manually calculating aggregate health status and health service delivery by age group and gender is eliminated because the system can automate these computations. While implementing electronic health records in Africa may seem far in the future, mobile phone data applications and OpenMRS makes it possible today and these electronic health records provide the foundation for patient messaging and reporting.

$\mathrm{MoTeCH}$ augments the individual level health record with information necessary for client messaging. For each client, data about registration status in the messaging program, phone\#s, who owns the phone, language, and time preferences for phone calls are maintained. Each client also has a unique seven digit system generated ID that is used by both the nurse and the client for identification purposes. Nurses use it to identify who is receiving care and clients use it when they call back into the MoTeCH system to retrieve their client-specific messages.

The most interesting (and challenging) software development activities were centered on two tasks: integrating the three major software applications and providing code to efficiently 
calculate when and where a message should be sent. While each of the three applications provides great support for tailoring the content of the application, in general they were missing the interoperable components that this project needed. Therefore, a considerable portion of our time was spent developing robust, fault-tolerant, code that wired the applications together. We expect that interoperability capabilities will improve in these software applications and we see considerable value in building these features into the applications.

Two specifications, a recommended care schedule and a messaging schedule, are used to determine when a message should be sent to a patient or a nurse. The recommended care schedule provides the timing for recommended service delivery, for example the schedule of immunizations for a child. The messaging schedule is independent of the care schedule which allows for variations in messaging timing based on the message recipient role. From these specifications, MoTeCH builds a calendar of upcoming events for each patient. When new data comes into the medical record, the schedules are updated for that patient if the information will alter the upcoming schedule. For example, if the first dose of tetanus is given, the second tetanus shot should be given at least four weeks from the date of the first dose. A periodic task in $\mathrm{MoTeCH}$ evaluates when a message should be sent from the messaging time specification and the calendar of upcoming events. The recommended care schedule and messaging schedule are defined using XML syntax with the goal of making it easy to use $\mathrm{MoTeCH}$ in other health domains.

There are a number of other, smaller, components of the $\mathrm{MoTeCH}$ application. Figure 3 illustrates some of the key components of the architecture: 
The Architecture of a Software System for Supporting Community-based Primary Health Care with Mobile Technology: The Mobile Technology for Community Health (MoTeCH) Initiative in Ghana

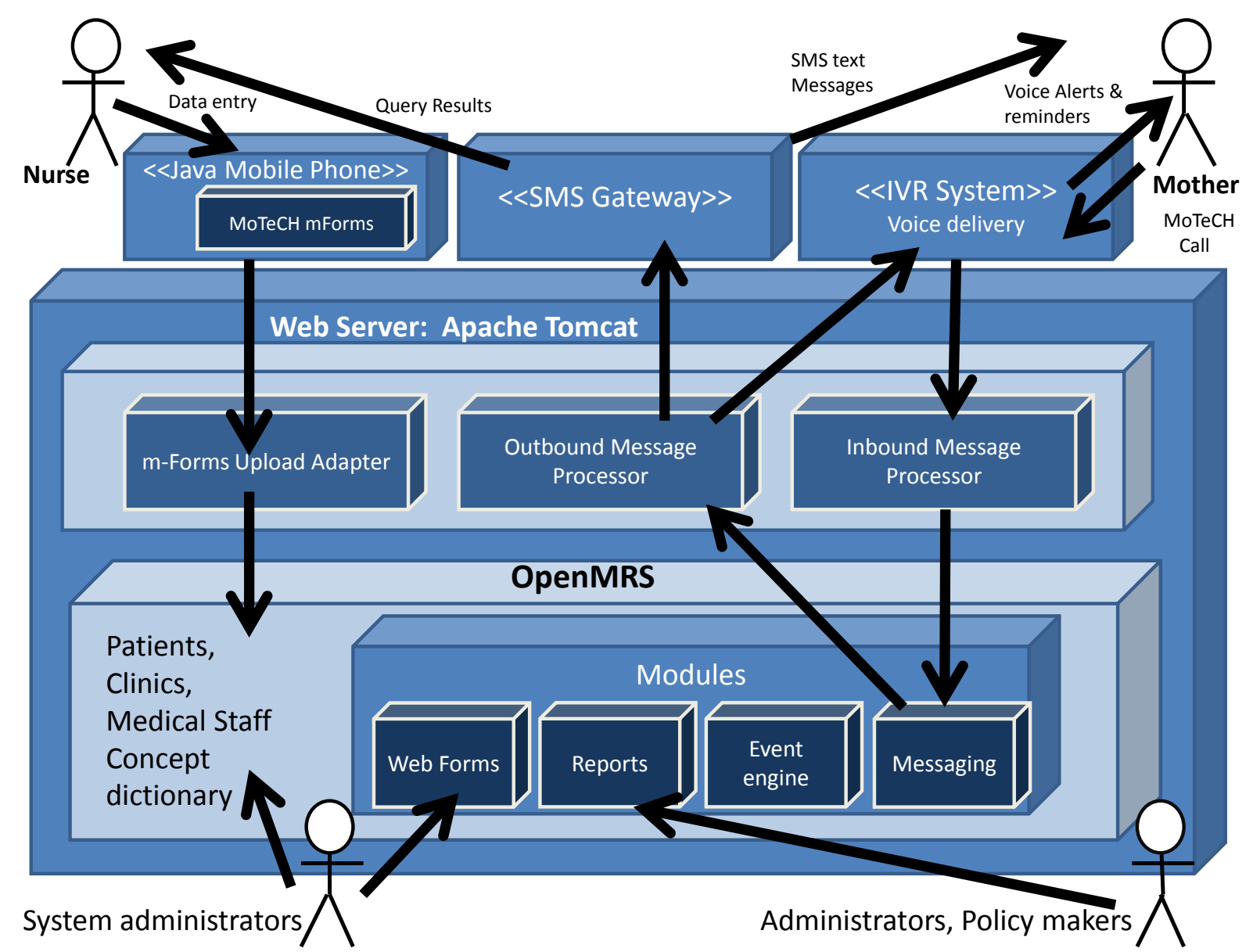

Figure 3: The Architecture of $\mathrm{MoTeCH}$

The extensible features of the electronic medical records system, OpenMRS, provided a solid foundation to build on. The OpenMRS concept dictionary allowed the project to combine wellestablished health definitions (anemia in pregnant women) with project specific data fields (i.e., patient telephone number). The extensible module architecture of OpenMRS allowed us to layer project specific logic onto the medical record system. We used this capability to develop $\mathrm{MoTeCH}$ modules for web based data management, nurse and patient messaging logic, an event engine that calculates a schedule of upcoming care for each patient, and administrative reporting.

We also added a small amount of logic to the core codebase of OpenMRS. The modification allowed for an efficient recalculation of upcoming care for a client when new service data about that patient was entered in the system. For example, when OpenMRS receives information about a child immunization, the timetable for upcoming immunizations for only this child is updated based on new data. In this respect, the open source nature of OpenMRS was critical to our project.

The mForms mobile data collection application noted in Figure 3 is built from OpenXData. This open source software includes a mobile client that runs on low-cost phones, a form designer, and server side data upload features. OpenXData implements a significant portion of the xForms 
The Architecture of a Software System for Supporting Community-based Primary Health Care with Mobile Technology: The Mobile Technology for Community Health (MoTeCH) Initiative in Ghana

standard which uses XML to represent the structure of data entry forms. This gave us the ability to quickly define and revise mobile data entry forms and played a critical role in delivering new functionality in a timely manner. We also modified the OpenXData application software to include the ability to report inconsistent data that could only be determined on upload to the server (for example, an invalid patient ID). Again, the ability to add functionality to existing application with many thousands of lines of code was critical to our success.

Figure 3 illustrates the data flows that $\mathrm{MoTeCH}$ software integrates into a cohesive system:

- Recording health service delivery: When a Nurse (upper right in diagram) provides care to a patient; this information is recorded in the appropriate form on the $\mathbf{m F o r m s}$ mobile phone application. When cell phone coverage is available, the nurse can choose to upload all stored forms to the server using the GPRS telecommunications standard. The Upload adaptor component parses the data, performs consistency logic, and notifies the client phone of errors associated with invalid forms. Invalid form entries are kept on the cell until the problem is resolved or the data is deleted. The Upload adaptor also converts the data into format appropriate to OpenMRS and updates the patient record. As previously mentioned, when this update is made a new schedule of upcoming care is recalculated based on the date of the new data.

- Messages sent to clients: When a client registers for MoTeCH messages, a calendar of expected care events are generated by the Messaging module. The Event engine module periodically checks this calendar to see if any clients are due for messages. This calculation is based on the due date and an evaluation of the medical record to determine if the service has been provided. Using declarative specifications (with XML syntax), $\mathrm{MoTeCH}$ will send messages to clients just before the health service (i.e. immunization) is due and continue to send messages for a designated period of time until the medical record system is notified that the service was delivered to the client. The language used for the message and the day and time the message is sent are based on patient preferences. Once the Event engine determines that a message should be sent to a client, it forwards the request to the Outbound message processor which holds the message until the correct time of day and then forwards the message request with the IntelliIVR voice application that plays the message to the client. If the call is not completed, then the message is stored to be sent again later. MoTeCH clients can also retrieve their messages at any time by contacting the system toll-free and then entering their ID number. Clients can listen to their messages as many times as they would like.

- Weekly Defaulters list is sent to nurses: The Event engine will also calculate, on a weekly basis, all clients who are overdue for health service in a particular nurse's catchment area and send this list to the Outbound message processor which then forwards the information to the nurse via an SMS Gateway. Nurses will receive these defaulter messages regardless of whether the client is registered to receive MoTeCH messages.

- Query support for nurses: Nurses can obtain information from $\mathrm{MoTeCH}$ via the query forms of $\boldsymbol{m F o r m s . ~ F o r ~ e x a m p l e , ~ a ~ n u r s e ~ m a y ~ b e ~ i n t e r e s t e d ~ a l l ~ p r e g n a n t ~ w o m e n ~ w h o ~ a r e ~ d u e ~}$ for an antenatal care visit in a particular village. The village name and type of service is entered into an $\boldsymbol{m F o r m s}$ form, uploaded to the mForms Upload Adaptor which then sends the request to the Messaging module. The Messaging module uses the Event engine to determine all clients who have defaulted on the designated health service in that CHPS zone 
The Architecture of a Software System for Supporting Community-based Primary Health Care with Mobile Technology: The Mobile Technology for Community Health (MoTeCH) Initiative in Ghana

and sends the results to the Outbound message processor which, in turn, sends the defaulter list to the SMS Gateway. The nurse will receive the results via SMS on their phone.

- District Health Information System Reports: Data from routine service delivery by nurses, sent from mobile phone data-entry forms and inserted into the client medical records is used to generate monthly reports on service provision.

i) Choosing a form

ii) Selecting a field

iii) Entering data

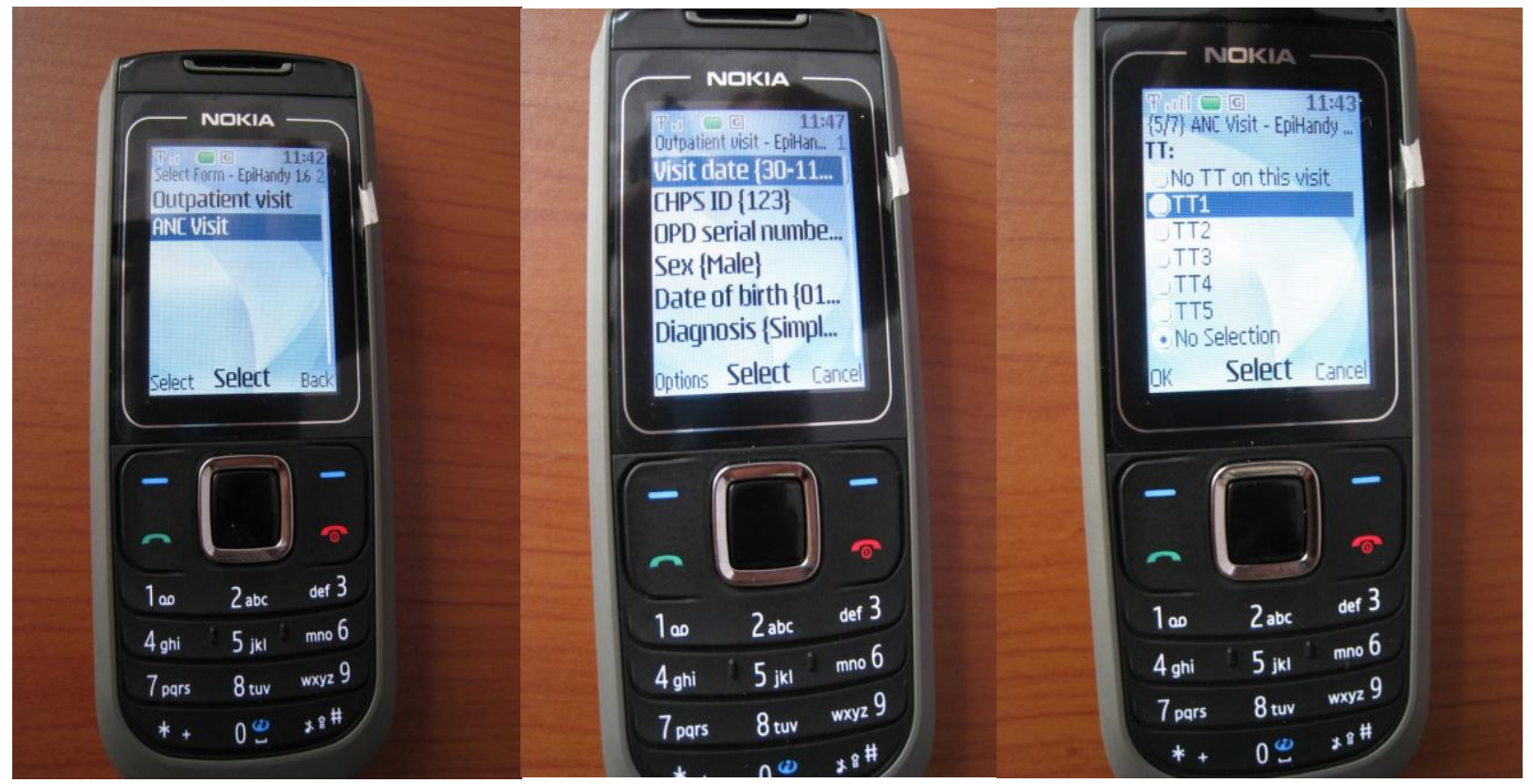

Figure 4: The Community Health Officer's MoTeCH data management sequence

By the end of 2011, the MoTeCH technical team had completed multiple iterations of deployment, testing, and revision so that the system was fully functional in one pilot district. Nonetheless, experience with the system has provided insights into ways that its architecture could be improved. In general, the interoperable software logic between the software applications should be less tightly coupled and the configuration of the system for new client messages, mobile data form content, and report generation require too much software developer expertise.

\section{Results}

\section{Initial lessons learned from implementation in the Upper East:}

Preliminary reactions from clients who have received $\mathrm{MoTeCH}$ messages have been mostly positive. Women have said that $\mathrm{MoTeCH}$ alerts informing them that they were approaching their expected delivery date allowed them to be more prepared when they began labor. Client interviews have also determined that $\mathrm{MoTeCH}$ informational messages promoting facility-based 
delivery and getting to a health facility as soon as possible after the onset of labor have influenced their delivery preferences and experiences. Some women have commented that these messages have empowered them to insist on accessing health services in ways that are sometimes discouraged by traditional beliefs and elder relatives. For example, one $\mathrm{MoTeCH}$ client said that during a previous delivery she labored at home for hours before going to a health facility, because elder female relatives said this demonstrates a woman's strength. But during her most recent delivery, after hearing $\mathrm{MoTeCH}$ messages, she insisted that she go to the nearest health facility as soon as she started feeling labor pains ${ }^{16}$.

Despite this evidence of success, $\mathrm{MoTeCH}$ has also encountered significant operational challenges. Since electrification has yet to reach most Kassena-Nankana villages, there are limited locations where telephones can be charged. Moreover, charging involves paying a fee to phone charging providers. For this reason, it is commonplace for users to keep their phones turned off until they need to make a phone call. Because cell phone use is a valuable commodity, gender problems that constrain women's access to familial resources apply as well to access to phones. Men and senior women in extended families are reluctant to lend phones to young women who might leave their phones on and consume battery power. Constrained access complicates $\mathrm{MoTeCH}$ initiation of the transmission of client messages. For the system to work, women must call the system to retrieve their messages. But, calling back into the system is not always easy for rural women who have little experience with navigating phone IVR routines. These challenges have been noted in other research projects and this is clearly an area where more research and investigation is needed ${ }^{17}$.

We are also in the process of developing a better understanding the operational challenges and benefits to frontline health workers. In some cases, better training is needed. For example, up until a couple of months ago, most community health volunteers were not reporting deliveries in the community. For the Community Health Officers (nurses), the extra burden of entering patient data needs to be weighed against the benefits of automated report generation. Some CHPS zones have been very diligent and proactive, but not all. More research, and quite possibly software refinements, is needed here as well.

\section{Evaluation Plan}

$\mathrm{MoTeCH}$ is a complex system with several different processes and engagement with multiple target groups. Understanding the impact of such a system requires a multi-dimensional evaluation approach utilizing quantitative and qualitative methods, examining the effects of the intervention on health workers, clients, managers, and policy makers. Columbia University's Mailman School of Public Health, in collaboration with the Navrongo Health Research Centre located in the Upper East, has designed an evaluation framework that seeks to understand the impact of the MoTeCH pilot on the health-seeking behavior by clients and the productivity, effectiveness, and the quality of data reported by health workers. Research also seeks to determine any challenges or barriers in using the system and any other unpredicted effects of the system on health workers and clients. 
The Architecture of a Software System for Supporting Community-based Primary Health Care with Mobile Technology: The Mobile Technology for Community Health (MoTeCH) Initiative in Ghana

\section{Discussion}

\section{Moving towards a more general health messaging system}

As $\mathrm{MoTeCH}$ became operational, we received requests to adapt the application to support data collection and messaging for other health problems and in other regions. Many of the key extensible elements are in place. OpenMRS supports the definition of new data concepts, location hierarchies, and health system providers. OpenXData has a form designer and data upload features that can be used without any custom programming. Finally, the design of the MoTeCH messaging uses an XML specification that allows users to declaratively specify which clients are eligible for a message and what the timing of those messages should be.

Still, there is much work to do before we can realize the vision of a health messaging system that will require little or no programming. Changes are needed in some of the components we use and in some of the software that resides at the interface of the larger components. These include:

- Use of an Open Source IVR system that uses the VXML (Voice XML) standard. The current use of a proprietary IVR system would require license charges and, quite possibly, customizations for new applications. Relying on the VXML standard for the MoTeCH IVR interface would allow multiple IVR solutions to be used in MoTeCH. Options include deploying a project-owned IVR system (for example, the open source Asterik/Voice Glue software, www.asterik.org and www.voiceglue.org, is VXML compliant) or using a IVR service provider like Voxeo (www.voxeo.org) that supports the VXML standard.

- An integrated MoTeCH content manager could help new projects to manage the message content that is sent to clients. The client content evolves through time and, currently, making changes requires software developer support. An integrated content manager would allow these changes to happen with programming.

- Improving the interface between cell phone data collection and OpenMRS. Currently, software needs to be written to map fields from mobile data collection forms to the OpenMRS data model. Ideally, this could be done without requiring software to be written. One possibility is to use a data integration tool, such as Mirth (www.mirth.org).

- The Event Engine module of MoTeCH may be better implemented with Rules Engine software. It is likely that new applications of $\mathrm{MoTeCH}$ will specify different schedules of health care and client messaging. Without a rules engine, this would require software development. Rule Engines generally have a mechanism to specify a collection of rules using a Graphical User Interface and collections of facts can tested against the rules. For MoTeCH, the client care history is a collection of "facts" that can be evaluated against the recommended schedule of "rules" for sending messages to the client. The open source jBoss project (www.jBoss.org) includes a combined workflow and rules engine that looks particularly appropriate for specifying the messaging sequence logic in $\mathrm{MoTeCH}$.

- The MoTeCH district reporting system is built from a large collection of custom coded SQL (Structured Query Language) statements. Evolving standards like SDMX-HD (www.sdmXhd.org) may provide a less complex specification system for data aggregation. 
The Architecture of a Software System for Supporting Community-based Primary Health Care with Mobile Technology: The Mobile Technology for Community Health (MoTeCH) Initiative in Ghana

- Analysis and a strategy for scaling up the system for a larger pool of users. Our current system is used for a population of approximately 74,000. We do not know what the limitations of the current system are, so an analysis is needed to identify some of the performance bottlenecks. The analysis would be the first step in identifying a strategy for scaling up to a national level.

Many of the above tasks are being addressed in an on-going MoTeCH re-engineering effort (http://code.google.com/p/motech/). The focus has been on developing support for larger populations, applying the ideas to different health systems, and developing a broader range of functionality with features such as pill reminder calls.

\section{Conclusions}

$\mathrm{MoTeCH}$ aims to address health information problems that are commonplace in Africa, where the flow of timely and accurate health information is often compromised by limited resources, households do not have formal addresses, and there are limited options for the distribution of quality health information. The application is addressed to the information needs of pregnant women and young mothers, frontline health workers, and district health managers. Pregnant women and young mothers can receive regular voice calls from $\mathrm{MoTeCH}$ that convey best health practices and remind of upcoming appointments. Nurses will enter client service delivery information into low cost mobile phones and this information will be used to automate the generation of monthly reports. The software architecture that provides this functionality is built by integrating well-tested applications for mobile phone-based data collection software, an electronic medical record system, and an Interactive Voice Response application.

$\mathrm{MoTeCH}$ is the initial step an iterative process of health information systems improvement in poor, rural regions around the world. Ultimately, we hope that providing timely and accessible health information to the key participants of the health care system will have a transformative impact on the effectiveness of health care delivery. While building these systems requires advanced software development skills, attention to standards based specifications and configurable design will make these systems more readily available to groups that can make use of them.

\section{Acknowledgements}

The MoTeCH pilot was funded by the Bill and Melinda Gates Foundation. MoTeCH is a project of the Ghana Health Service (GHS). MoTeCH would not have been possible without the leadership and contribution of Drs. Frank Nyonator, Director of Policy Planning Monitoring and Evaluation Division of the GHS and the full support of the district health management team and frontline workers in Kassena-Nankana West District of the Upper East Region. The OpenMRS and OpenXData open source software communities not only provided well-tested source code, their advice and encouragement during the year and half of $\mathrm{MoTeCH}$ development was invaluable. We are extremely grateful for all of the support and we hope that these communities can share in any success we may have. 
The Architecture of a Software System for Supporting Community-based Primary Health Care with Mobile Technology: The Mobile Technology for Community Health (MoTeCH) Initiative in Ghana

\section{Corresponding Author}

Bruce MacLeod

Mailman School of Public Health and the Computer Science Department of the University Southern Maine

96 Falmouth Street

Portland, Maine 04104

Phone : 2077804285

Email: macleod@usm.maine.edu

1. Perry H, Freeman P, Gupta S, Rassekh BM. "How effective is community-based primary health care in improving the health of children?" Report of the Community-Based Primary Health Care Working Group, International Health Section, American Public Health Association, Franklin West Virginia: Future Generations. 2009

2. Earth Institute of Columbia University Technical Task Force on Community Health Workers. One Million Community Health Workers. Technical Report of the Earth Institute: New York: Columbia University. 2011

3. International Telecommunication Union. "Global mobile statistics 2011," in the unpublished web report Mobithinking. http://mobithinking.com/mobile-marketing-tools/latest-mobile-stats. 2011

4. Kwapong OATF. 2008. The health situation of women in Ghana. Rural Remote Health. 8, 963. http://www.rrh.org.au.

5. Ngom P, Debpuur C, Akweongo P, Adongo P, Binka FN. 2003. Gate-keeping and women's health seeking behavior in Navrongo, northern Ghana. Afr J Reprod Health. 7(1), 17-26. http:// dx.doi.org/10.2307/3583341

6. Snow RW, Omumbo JA. "Malaria." Chapter 14 in Jamison DT, Feachem RG, Makgoba MW, et al., editors. Disease and Mortality in Sub-Saharan Africa. 2nd edition. Washington (DC): World Bank; 2006.

7. Binka, Fred N. Fighting malaria in Africa, The Lancet, 371(9622):1409. 26 (April) doi: 10.1016/S0140-6736(08)60614-5.2008

8. Adongo P, Phillips JF, Kajihara B, Fayorsey C, Debpuur C, et al. 1997. Cultural factors constraining the introduction of family planning among the Kassena-Nankana of northern Ghana. Soc Sci Med. 45(12), 1789-804. http://dx.doi.org/10.1016/S0277-9536(97)00110-X 9. Adongo PB, Phillips JF, Binka FN. 1998. The influence of traditional religion on fertility regulation among the Kassena-Nankana of northern Ghana. Stud Fam Plann. 29(1), 23-40. http:// dx.doi.org/10.2307/172179

10. Binka FN, Bawah AA, Phillips JF, Hodgson A, Adjuik M, et al. 2007. Rapid achievement of the child survival Millennium Development Goal: Evidence from the Navrongo Experiment in northern Ghana. Tropical Disease and International Health. 12(5), 578-93. http:// dx.doi.org/10.1111/j.1365-3156.2007.01826.x 
The Architecture of a Software System for Supporting Community-based Primary Health Care with Mobile Technology: The Mobile Technology for Community Health (MoTeCH) Initiative in Ghana

11. Frimpong J, Helleringer S, Awoonor-Williams JK, Yeji F, Phillips JF. 2012. Does supervision improve health worker productivity? Evidence from the Upper East Region of Ghana. Trop Med Int Health. (forthcoming).

12. Nyonator FK, John KAW, Phillips JF. "Scaling down to scale up: Accelerating the expansion of coverage of community-based health services in Ghana." Paper presented at the International Conference on Family Planning, Dakar Senegal, November 29-December 2, 2011. 13. International Telecommunication Union. "ICT Statistics Database, Mobile cellular subscriptions", http://www.itu.int/ITU-D/ICTEYE/Indicators/Indicators.aspx\# 2011 14. World Development Indicators Database, The World Bank Group. (Mobile Cellular subscriptions per 100) http://data.worldbank.org/indicator/IT.CEL.SETS.P2) 2011 15. Live MW. GSM coverage map : Ghana (http://maps.mobileworldlive.com/network.php? cid $=134 \&$ cname $=$ Ghana) 2011

16. Awoonor-Williams JK, Phillips JF, Stone A, Helleringer S, MacLeod B, et al. "The Mobile Technology for Community Health (MoTeCH) Initiative: An m-Health System Pilot in a Rural District of Northern Ghana." Paper presented at the Annual Conference of the American Public Health Association, Washington, DC, October 30-November 3, 2011.

17. Haberer JE, Kiwanuka J, Nansera D, Wilson IB, Bangsberg DR. 2010. Challenges in using mobile phones for collection of antiretroviral therapy adherence data in a resource-limited setting. AIDS Behav. 14, 1294-301. http://dx.doi.org/10.1007/s10461-010-9720-1 